\title{
Aprender A Trabajar En Equipo: Clave De las Organizaciones Que APREnden
}

\author{
Julio Andrés Palomino Silva \\ Facultad Ciencias Contables de la UNMSM \\ Lima - Perú
}

\begin{abstract}
RESUMEN
- El creciente grado de competitividad Empresarıal ha provocado un vuelco en las estrategias clasıcas de adaptacion externas y también en las diferentes formas de organización interna dentro de la empresa, obligando a estas a adaptarse a nuevas formas organizativas. La principal forma de hacer frente a estos retos lo encontramos en aprender a atrabajar en equipo para llegar a la consecución de resultados.
\end{abstract}

Palabras clave: Unidades organizativas, liderazgo, gestión de resultados, estrategıa organizacional.

\section{INTRODUCCIÓN}

La disposición de lo económico y social en sistemas cerrados y estables produce situaciones de baja competencia ínter empresarial y como consecuencia de ello, culturas de empresa donde prevalecen convicciones que defienden estructuras y comportamientos rígidos, el inevitable paso hacia sistemas económico y sociales abiertos e inciertos esta favoreciendo en las empresas la necesidad de abrir y agilizar sus diseños organizativos y las aportaciones de las personas a los proyectos empresariales.

Esas necesidades que las empresas tienen por dar respuestas acertadas en forma de servicio o productos allí donde concurren viene determinada siempre por dos variables básicas e interdependientes: por su capacidad para adaptarse a las exigencias externas y también en las diferentes formas de organización interna que tradicionalmente las empresas han dispuesto.

Efectivamente, estamos asistiendo al fin de las estructuras estáticas y jerarquizadas que bajo distintas denominaciones se han caracterizado por la fragmentación y segmentación de la empresa en unidades funcionales que en términos generales han tendido ha alejarse entre ellas mismas y en relación a la estrategia general. Esta situación esta lógicamente cambiando y podemos observar como están apreciando nuevas formas organizativas caracterizadas por su flexibilidad, rapidez y descentralización. La organización ya no es, en este sentido, estructura ni organigrama, es sobre todo menor coste y facilitación.

\section{LAS UNIDADES ORGANIZATIVAS COMO EQUIPOS DE TRABAJO}

En este nuevo escenario las unidades organizativas de las empresas se convierten así en pequeñas organizaciones que explican su razón de ser y su madurez en la organización, en la medida que son capaces de identificar y aportar determinado valor al conjunto de los procesos y resultados globales esperados de una empresa. Esta misión que se le asigna hace especialmente necesario que cada unidad aprenda a trabajar y a desarrollarse como un verdadero equipo 
de trabajo. Desde esta consideración es seguro que el trabajo en equipo es lo que probablemente va a explicar que las unidades organizativas se constituyan como tales.

Además, en la situación ya apuntada de flexibilidad, aparecen de forma paralela a la estructura formal de una empresa, unidades de trabajo conocidas como equipos ad-hoc, equipos de proyectos de mejora multidisciplinares, etc. Que se forman con el objeto de lograr determinados resultados que no se contemplan bajo cierta estandarización y que desaparecen cuando han logrado alcanzar los objetivos sobre los que deben trabajar. De esta forma, una misma persona tiene la posibilidad de participar en varios equipos a la vez, lo que conlleva la circulación abierta de personas por la organización y la adscripción simultanea a varias unidades. Estas nuevas posibilidades organizativas van a cambiar de manera significativa muchos de los aspectos en los que se han basado las relaciones entre directivos; colaboradores. Entendemos que una de las consecuencias mas interesantes de ese cambio por parte de los que desempeñen tareas directivas, va ser la oferta interna de proyectos profesionales a los que podrán optar colaboradores en función de lo que previsiblemente sean capaces de aportar en forma de valor añadido. Pero también en ese contexto de mayor exigencia aparece bajo cierta paradoja la elección de directivos por parte de los colaboradores, surge de esta forma el «quiero ser dirigido por ti» para tal proyecto de trabajo. En una primera valoración de lo que esto supone, no es difícil imaginar una función directiva entendida sobre bases de proceso y potencial de influencia sustancialmente diferentes a las hasta ahora convencionales, pero sobre todo, una dirección de personas con mayores posibilidades, que recogerá nuevos y mas apasionantes retos basados en la credibilidad y el reconocimiento abierto hacia su función. Esas nuevas concepciones encierran cambios sobre los que se van a asentar las relaciones dirección- colaboración de cualquier organización en un futuro inmediato.

Nos encontramos delante de dos tipos de unidades-equipos de trabajo que se complementan y se enriquecen. Por un lado, unidades organizativas formalizadas y relativamente pequeñas, con capacidad de respuesta rápida y fuertemente conectadas a la estrategia global. Y por otro, unidades paralelas y añadidas que se crean para dar respuesta a necesidades concretas y que desaparecen con el cumplimiento de su encargo.

Entendemos que ambos tipos de unidadesequipos que van a convivir de forma complementaria y necesaria, mantienen en común dos tipos de consideraciones; una la necesidad de vincular su actividad a la consecución de resultados para clientes internos o externos, y otra el reconocimiento de que el logro de esos resultados se correlaciona con determinados factores que favorecen el éxito de los mismo. Esa doble dimensión y exigencia es precisamente la esencia de lo que se entiende como un equipo de trabajo, es decir la necesaria interdependencia entre unos indicadores concretos de resultados que deben conseguirse en el marco de las unidades organizativas y unos determinados factores del propio equipo que decididamente los favorecen.

Desde esa concepción queremos abordar como las unidades organizativas deben aprender a trabajar en equipo y como pueden $y$ deben contribuir a que una organización 
posea en su conjunto capacidad de aprendizaje.

\section{LOS RESULTADOS DE LAS UNIDADESEQUIPOS}

Cualquier unidad organizativa debe plantearse que la misión básica pro la cual existe es la consecución de resultados. A pesar de su obviedad nos atrevemos a formularlo al revés, es la consecución de determinados resultados lo que va a favorecer que exista una unidad organizativa. Para cualquier organización el conjunto de resultados puede estar relacionados, dependido de la actividad que desarrolle como por ejemplo, volumen de ventas, rendimiento de productividad, márgenes de aportación, cumplimiento de determinados índices de calidad, diferenciación o la rapidez de atención a los clientes. De lo que se deduce que los resultados de las unidades equipos son las síntesis que responde a alguna de las siguientes cuatro estrategias básicas o a varias de ellas a la vez: nos referimos a la cantidad, al coste, a la calidad y al tiempo, más exactamente, los resultados siempre se espera que se conviertan en el aumento de la cantidad, en la disminución del coste, en la mejora de la calidad y en la reducción del tiempo. Esas cuatro variables alimentan al conjunto de indicadores posibles que se esperan de las unidades organizativas. Se trata pues de fijar donde se deben alcanzar resultados, cuales son los que se espera que se logren y como sabremos que lo hemos logrado con éxito.

Cuando se especifica que resultados concretos se esperan de un departamento, una sección, un área o un comité de dirección, se enmarcan las variables que en términos de cantidad. Coste y tiempo esa unidad organizativa debe conseguir para un periodo de tiempo y, también la forma de conocer sus logros, mediante indicadores que los expliquen y que, ofreciéndose a los clientes, internos o externos de los mismos estén inequívocamente interesados porque dependen en alguna forma de ellos.

Cuadro 1

\section{LOS RESULTADOS COMO CONSECUENCIA DE LA INTERDEPENDENCIA ENTRE UNIDADES Y EQUIPO DE TRABAJO}

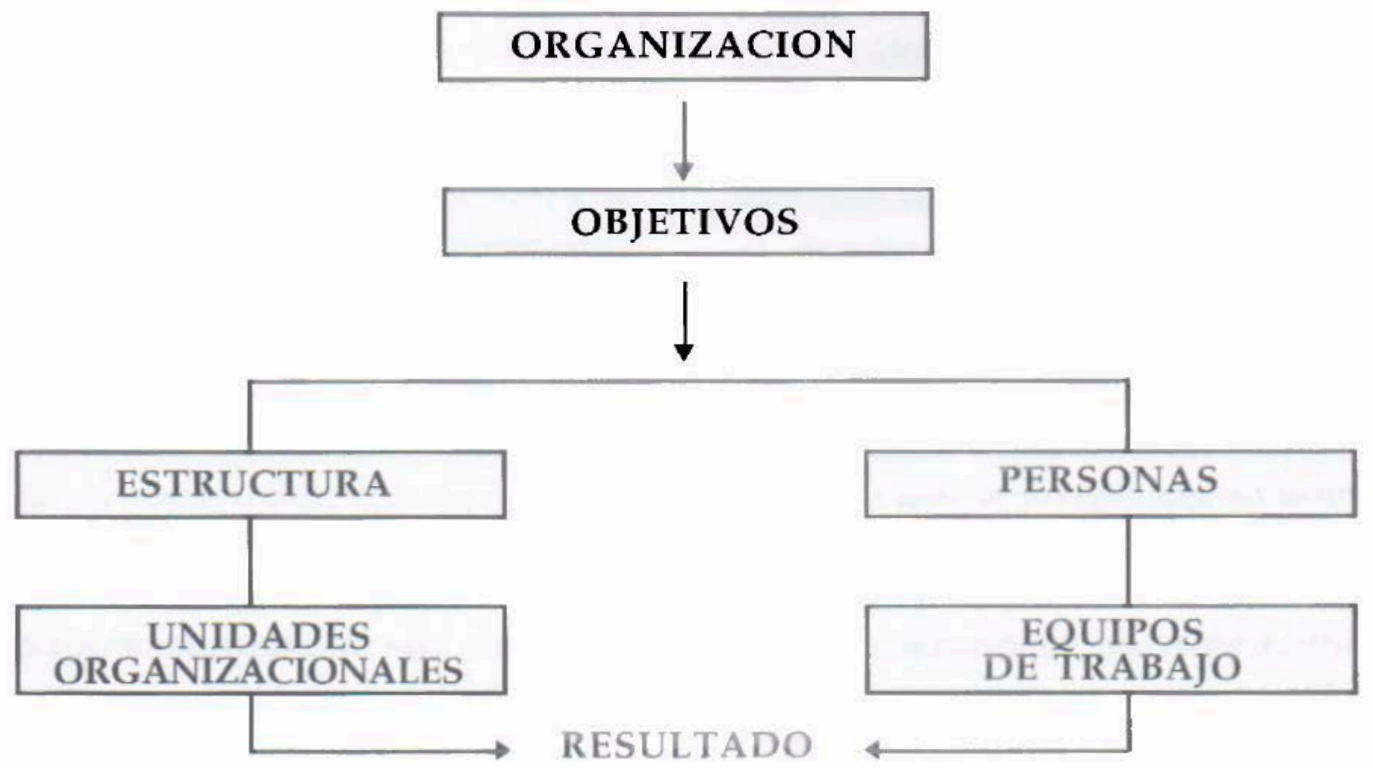




\section{LOS FACTORESQUE FAVORECEN EL LOGRO DE RESULTADOSDE LA UNIDAD-EQUIPO}

A lo largo de los últimos años hemos recogido las opiniones que nos han manifestado integrantes de varios equipos de trabajo de diferentes organizaciones empresariales. Se les ha preguntado, a partir de sus experiencias formativas $y$ profesionales, cuales son los factores que favorecen el logro de resultados, es decir que cosas ocurren en sus equipos en función de su experiencia cuando consiguen sus resultados y por tanto que características ayudan al éxito en sus respectivos equipos de trabajo. El conjunto de respuestas las hemos agrupado en base a los factores mas saltantes cuando sus unidades de trabajo consiguen un resultado de éxito. Presentamos a continuación un conjunto de factores que se han constituido como aquellos de mayor significación.

1. Identidad de Equipo: Este primer factor explica y responde con efectividad a preguntar como quienes somos como unidad en la organización, como nos definimos, quienes somos como unidad en la organización, como nos definimos, quienes somos, que nos diferencia de los demás y cual es nuestra misión, por tanto para que estamos. Este factor enfatiza la importancia de la identidad grupal e interioriza y configura el sentido que el equipo tiene para los individuos y para la organización. Se puede resumir este factor definiéndolo como aquel que determina una forma de ser del equipo unidad.

2. Objetivos: Este factor por su obviedad pudiera quedar sin ser considerado, nos referimos a que el equipo tenga lógicamente resultados que obtener: queda claro que una unidad de trabajo que no tenga un conjunto de resultados que obtener difícilmente va a conocer si tiene éxito. Aquí el indicador y el factor son dos caras de un mismo hecho, especificar los resultados que deban conseguirse y que el equipo perciba y trabaje orientando su actuación hacia los resultados que tiene que obtener son, respectivamente un fin $y$ un medio para conseguirlo. La cuestión formulada en términos de que debemos hacer explicita este segundo factor.

3. Criterios de Actuación: En este factor se agrupan aquellos conceptos que indican como se han de hacer las cosas Los criterios de actuación se alimentan de determinados principios, creencias $\mathrm{y}$ convicciones que son las formas de entender como hacemos nosotros las cosas. De igual forma que en el primer factor, se trata en este, de establecer una determinada manera de hacer del equipo unidad. En este caso el factor se concreta en como hemos de hacer aquello que debemos.

4. Liderazgo que se adapte y facilite el cambio: Una dirección del equipo con una doble perspectiva, por una lado, con estilo de liderazgo capaces de adaptarse y dar respuesta a las diferentes situaciones de los colaboradores, posibilitando su crecimiento profesional. Y por otro, con voluntad decidida de transmitir, de transformar, de cambiar sobre todo el comportamiento de los colaboradores. Un liderazgo que descentralice la estrategia, capaz de traducir mediante su influencia, los estratégico y global a lo operativo y concreto. Una dirección que interprete con acierto que ocurre tanto fuera como dentro de la empresa y que sepa transmitirlo y proyectarlo a su equipo.

5. Toma de Decisiones Efectivas: Con 
proceso de decisión claros que permitan conocer los mecanismos de decisión que vinculen al equipo y que orienten esas decisiones hacia los resultados que se esperan del equipo. El conocimiento de los procedimientos de decisión permite a la unidad organizativa centrar su actividad y esfuerzos hacia aquello que realmente es importante.

6. Alta disposición favorable de los integrantes: El comportamiento de personas motivadas e ilusionadas con lo que hacen, favorece decididamente la consecución de resultados. Un equipo involucrado y comprometido hacia un proyecto común configura un factor muy ampliamente considerado y deseado.

7. Actividad Orientada hacia el Exterior: El objeto de la actividad del equipo, es decir, aquello que hace en forma de productos o servicios, debe diseñarse y desarrollarse en función de la satisfacción de unos usuarios o clientes a los que están dirigidos. También aquí se incluye las relaciones de colateralidad hacia su entorno inmediato que articulen de forma satisfactoria al equipo con otras unidades de la misma organización.

8. Competencias Profesionales: La preocupación por detectar e incorporar de forma constante a personas con capacidades, conocimientos, aptitudes, creatividad, iniciativa, talento y potencial, tanto en la propia organización como fuera de ella. El conjunto de elevadas competencias de las personas se manifiesta como un factor clave de éxito.

9. Gestión de Resultados y Desempeños: Con sistemas que con objetividad gestionen los indicadores de resultados y también la forma en que se consiguen. Que permitan reconocer a las mejores aportaciones y corregir a las que puedan $v$ deban mejorar. Que la evaluación de la contribución de los integrantes no se situé en un nivel de desconocimiento y subjetividad que pueda provocar arbitrariedad y partidismo.

10.Aprendizaje Permanente: Favorece y estimula el aprendizaje continuo y compartido de unos con otros. Posibilitando el crecimiento profesional de las personas en particular y del equipo en general. Con proceso de aprendizaje que permitan mejorar los conocimientos, las actitudes y los comportamientos. Que permitan mejorar los conocimientos, las actitudes y los comportamientos, desarrollándose en el marco del equipo oportunidades de optimización y mejora.

11. Organización del Trabajo: Una disposición de los procesos y de las personas diseñada para facilitar la significación y la identificación con la actividad que se realiza. Que incorpore el para que se esta haciendo y que de sentido al que hacer diario. Con una organización que articule la participación, el enriquecimiento del trabajo y posibilite la generación del valor añadido a través de la flexibilidad y polivalencia de sus integrantes.

12. Compensación Satisfactoria: Que no existan desequilibrios, arbitrariedades ni privilegios en la retribución de los integrantes del equipo. Que se perciba una retribución que de respuesta a un adecuado grado de equidad interna entre los individuos, dando a contribuciones parecidas compensaciones semejantes. Que en la comparación hacia el exterior se perciba la compensación competitiva. ajustada respecto a puestos y trabajos comparables y también que se responda adecuadamente al principio de compensar más al mejor.

13.Conflictos como Oportunidades: Dona 
las diferencias se tratan abiertamente sin reservas injustificadas que esconden y retarden los problemas. En la confrontación de intereses debe buscarse la ganancia compartida y la resolución del conflicto favorece la consecución de nuevos niveles de mejora y oportunidad no considerados hasta entonces. Generar confianza en el dialogo frente a la confrontación, sabemos que a mayor grado de implicación, menor grado de conflicto.

A través de la exposición de cada uno de estos factores se han resumido en forma de comportamientos de equipos aquellos factores que mas se han manifestado como favorecedores y desencadenantes de la consecución de los resultados en las unıdades organizativas. A bien seguro que se les podrían añadir algunos más que, sobre todo, en cada situación empresarial deberán adquirir la relevancia que su contexto explicaría. Nos parece que en términos generales recogen los aspectos más significativos por los que los equipos pueden conseguir para lo cual se explica su razón de ser.

En nuestra observación del trabajo en equipo hemos entendido que a menudo se producen dos errores importantes que tienen relación entre si. El primer error es el centrar la actividad y los esfuerzos del equipo únicamente en la consecución de resultados, no considerando los factores que decididamente ayudan a conseguirlos y no fomentando, en especial manera desde la dirección que para conseguir los resultados esperados es preciso facilitar que se produzcan determinados factores. El segundo error supone lo contrario del anterior nos referimos a considerar la misión del equipo a través de los factores de éxito sin conectarlos a resultados que expliquen su contribución organizativa. De estas dos prácticas erróneas se desprende la necesidad de interdependencia, el éxito de un equipo desde ese punto de vista es la síntesis de dos dimensiones necesarias $y$ complementarias.

\section{EL DESARROLLO DE LAS UNIDADES EQUIPOS}

Como consecuencia de lo que hemos considerado hasta este momento, proponemos cuatro pasos que formulamos en forma de reflexión deben ayudar al desarrollo de las unidades equipos de trabajo en un contexto de aprendizaje organizacional.

A. Identificación de la Estrategia Organizacional

- En los próximos meses/años que resultados se van a precisar que consiga la empresa.

- Cuales van a ser los resultados prionitarios de la organización y que conjunto de resultados van a ser secundarios.

- Donde vamos a tener que centrar nuestros esfuerzos estratégicos.

B. Especificación de los Resultados para la Unidad equipo.

- En su conjunto cuales van a ser los resultados que conectados a los globales, se espera que consiga cada unidad.

- Cual es el nivel de logro de determinados resultados hasta ese momento.

C. Diagnostico de los Factores de Éxito del Equipo.

- Cuales son los factores que mejor favorecen el éxito en nuestro equipo.

- Como se posiciona el equipo, respecto a los dieciséis factores de éxito propuestos.

- Que podemos hacer para mejorar estos factores.

D. Planes de Actuación

- En que planes vamos a centrar los esfuerzos de mejora de resultados del equipo.

- Cuales son los resultados sobre los que se va a trabajar prioritariamente en los próximos meses/años

- Como vamos a saber que se han logrado 


\section{LAS CUATRO FASES EN EL DESARROLLO DE LAS UNIDADES EQUIPOS DE TRABAJO}

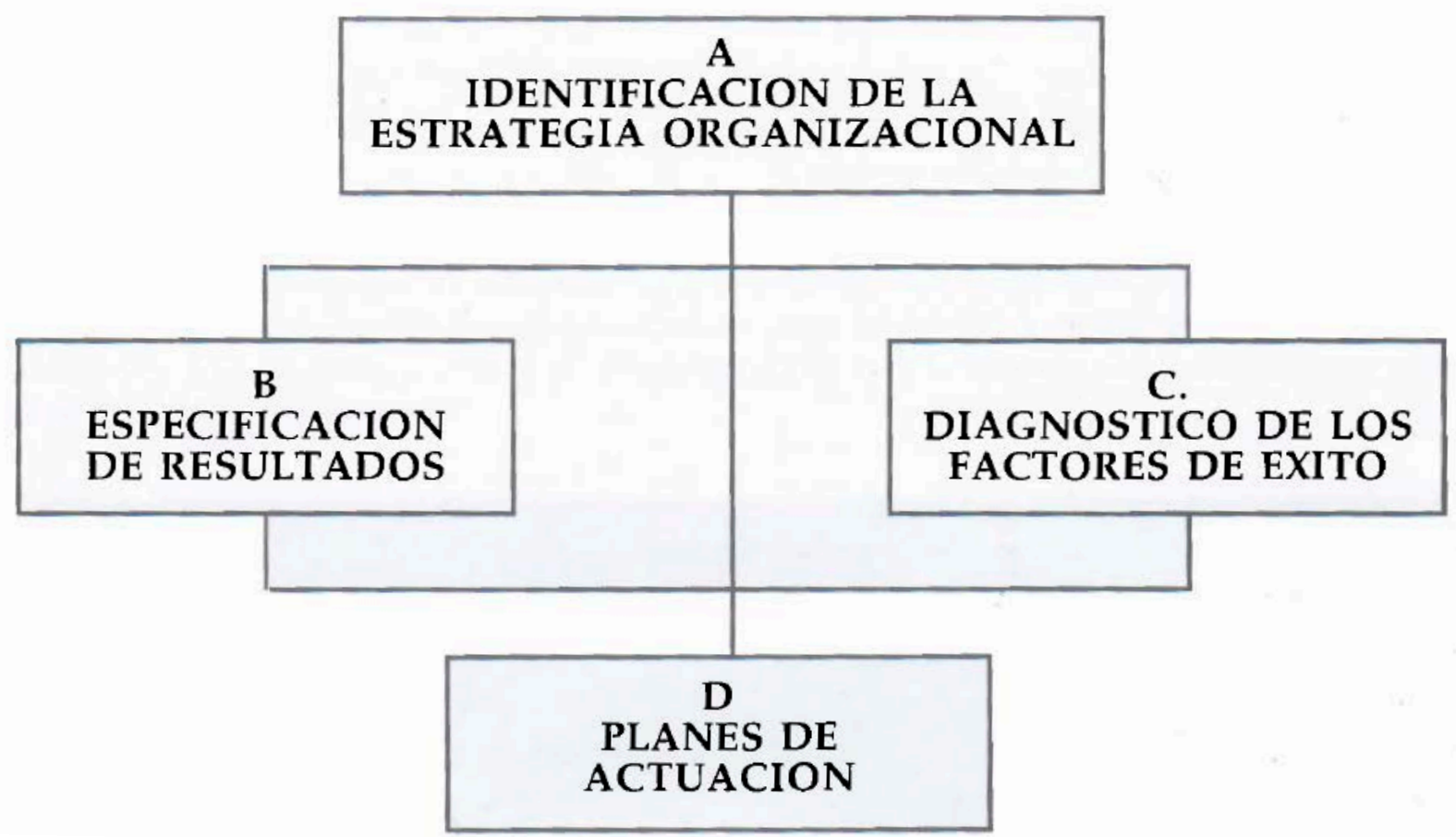

El trabajo sobre las respuestas a estas cuestiones favorece con toda seguridad el desarrollo de las unidades equipos de trabajo. El trabajo en equipo en ciertas forma, es todo aquello que justifique que el resultado de su actividad será siempre de mayor valor que la suma de cada uno de los componentes de la unidad organizativa.

Una ultima reflexión por que las organizaciones deben ser capaces de aprender y por extensión para que necesitan aprender las unidades que las componen. $\mathrm{La}$ sencillez de la respuesta no debiera hacer pensar en su simplicidad. El cambio permanente es la convivencia con la incertidumbre y es probablemente el aprendizaje aquello que nos proporciona la posibilidad de ganar certeza a la complejıdad, al tiempo que nos permite tener en el marco de las organizaciones la oportunidad para disfrutar de experiencias que nos deben conducir sobre todo a hacer y ser algo mejores.

\section{REFERENCIAS:}

1. Bedoyre, $\mathrm{O}$, como resolver problemas en grupo. Ed. Grafica.

2. Blake, R, Mouton, J y Allen, R. El trabajo en Equipo. ¿Qué es y como se hace?

Ed. Deusto. Madrid, 1990.

3. Dyer, W.G. Formación de Equipos. Ed. Addison - Wesley 1987

4. Lattman, Ch.y Garcia Echevarria, S: Management de los Recursos Humanos. Ed. Días de Santos. Madrid, 1992.

5. Paul, A Creatividad y grupos Pequeños. Ed. Pirámide. Madrid, 1985.

6. Reddin. B. y Ryan, D: Manual de Dirección por Objetivos. Ed. Deusto, Bilbao, 1993.

7. Rodríguez Porras, J. M:La Participación y la Calidad Integral. Ed. Deusto Bilbao, 1991

8. Shein,E.HyBennis, WG:El Cambio Personal y Organización a Través de Métodos Grupales. Ed. Herder, Barcelona, 1980. 